\title{
Der Partizipationsmythos „Partizipation bedeutet, alle immer an allem zu beteiligen“"
}

\author{
Andreas Seebacher, Sophia Alcántara \& Alexandra Quint
}

Ein Partizipationsmythos ist ein Komplex von individuellen und kollektiven Vorstellungen über Partizipation, die sich unter den Akteuren eines Reallabors als unhinterfragte Erwartungshaltung hartnäckig halten und-ohne ausreichende Evidenz - Entscheidungen (mit) beeinflussen. Ein solcher Mythos kann entweder bereits bei Beginn der Projekttätigkeit in den Köpfen der Beteiligten vorhanden sein, er kann während der Implementierung entstehen, oder er kann von außen an ein Reallabor herangetragen werden.

In der Reallaborarbeit hält sich der Mythos, eine permanente und allumfassende Beteiligung aller Praxisakteure sei ein besonderes Qualitätsmerkmal. Entsprechend wird die Qualität der Reallaborarbeit und ihrer Ergebnisse an der (Nicht-)Erfüllung dieses Anspruchs gemessen - und in der Öffentlichkeit kritisch beäugt. Im Tun von Reallaboren kann sich der Anspruch, alle immer an allem zu beteiligen, in unterschiedlichen Phasen manifestieren: beispielsweise in den frühen Phasen der Reallaborarbeit, wenn es darum geht, die inhaltliche Ausrichtung zuzuspitzen, oder in den späteren Phasen, wenn es darum geht, zu entscheiden, wie und wann Praxisakteure in die Forschungsaktivitäten eingebunden werden sollen. Der umfassendste Anspruch ist eine phasen- und aktivitätsübergreifende Partizipation aller Akteure.

In der Praxis zeigt sich jedoch, dass intensive Partizipationsangebote, die sich über die gesamte Laufzeit erstrecken und an die gleichen Akteure richten, zur Überanstrengung der Ressourcen der Praxisakteure führen können. Abflachendes Interesse an der Mitgestaltung und reduziertes Engagement bis hin zum Rückzug aus dem Prozess sind mögliche Effekte dieser Überforderung. Dieses Phänomen kann in besonderem Maße auf ehrenamtlich tätige Praxisakteure zutreffen und als eine Art Teilhabemüdigkeit bezeichnet werden (vgl. zu einer solchen „Beteiligungsmüdigkeit“" auch Eckart et al. 2018).

Ehrenamtlich Tätige beachten das Verhältnis von anstehenden Aufgaben und ihrer verfügbaren (Frei-)Zeit oft sehr genau und handeln entsprechend. Und dasselbe gilt für das Verhältnis zwischen Aufwand und Ertrag. So konnte beispiels- 
weise im Zuge des sogenannten BürgerForums des Reallabors 131: KIT findet Stadt (BaWü-Lab R131) festgestellt werden, dass die Praxisakteure erwarteten, dass ihre Anregungen bald in konkretes Handeln umgesetzt würden bzw. dass ihre tatkräftige Mitarbeit beim Anschieben von Projekten Wertschätzung erfahren würde. Hierin liegt eine große Herausforderung für das Format Reallabor, will es nicht in den Geruch kommen, bloßes „Particitainment“ zu bieten, wie Selle (2011) das für Bürgerbeteiligungsverfahren im Rahmen von Stadtentwicklungsprozessen diagnostiziert hat. Dies könnte nämlich eine „Inflationierung (...) wirkungsloser Teilhabe-Verfahren“ provozieren und „womöglich Politik- und Planungsverdrossenheit befördern" (ebd., S. 3), gegen die Partizipation - auch die in einem Reallabor - eigentlich ein Mittel sein soll(te). Es gilt also, darauf zu achten, Partizipation dort vorzusehen, wo sie eine benennbare Wirkung haben kann und soll - und nicht als Selbstzweck.

Aus Sicht der Wissenschaftler(innen), die ein Reallabor leiten und Partizipationsangebote an die Praxisakteure machen, muss der Anspruch einer permanenten und allumfassenden Beteiligung aus weiteren Gründen auf den Prüfstand gestellt werden: Zum einen kann die Überanstrengung auch sie selbst treffen und dazu führen, dass sie sich bei ebenfalls limitierten Ressourcen nicht auf ihre zentrale Aufgabe des Forschens zu konzentrieren vermögen. Zum anderen droht ihnen die Gefahr der Frustration, wenn sie sich womöglich damit auseinandersetzen müssen, dass das Interesse der Praxisakteure an Partizipation nicht so groß ist, wie sie das gemäß dem partizipativen Ideal des Reallaborformates gerne hätten.

Die Erfahrungen der Arbeiten in den BaWü-Labs ${ }^{1}$ beider Förderlinien haben gezeigt, dass die Ansprüche gerade bei zivilgesellschaftlichen Akteuren sehr unterschiedlich sind: Diese reichen vom Wunsch nach einer möglichst umfassenden Beteiligung, inklusive der Teilnahme an (wissenschaftlichen) Projekttreffen, bis hin zu einer nur sehr punktuellen, nur für sie selbst relevanten Einbindung in einem konkreten Realexperiment zu einem sehr spezifischen Thema (beispielsweise zu urbanem Gärtnern und Imkern, wie es in einem der Realexperimente des BaWü-Labs R131 bearbeitet wurde). Eine weitere Beobachtung ist, dass der Wunsch nach umfassenden Beteiligungsangeboten bei einigen Akteuren anfangs sehr groß ist und später von der Erkenntnis abgelöst wird, solche Angebote mangels Zeit nicht in vollem Umfang wahrnehmen zu können.

Demgegenüber besteht ein Zusammenhang zwischen dem Erfolg eines Reallabors, der darin realisierten Partizipationsqualität und der langfristigen Perspektive der Beteiligung, weil ein kontinuierlicher Partizipationsprozess das Mitwirken der oft ehrenamtlich tätigen Akteure befördert und die Qualität der partizipa-

1 In Baden-Württemberg wurden ab 2015 in den zwei Förderlinien „Reallabore“ und „Reallabore Stadt“ insgesamt 14 Reallabore (die „BaWü-Labs“) gefördert. 
tiv gewonnenen Ergebnisse steigert. Arbeiten im On-off-Modus mit langen Partizipationspausen funktioniert im Reallabor nicht: dieses ist auf Co-Design und CoProduktion ausgelegt und dazu bedarf es einer soliden, durch konsistenten und permanenten Dialog ermöglichten Vertrauensbasis.

Bei der Planung der Partizipation in einem Reallabor sind demnach vorab einige Fragen zu beantworten, deren Klärung bei der Akzentuierung und Rhythmisierung von Beteiligung helfen kann, wie z. B.: Wer sind die für das Reallabor relevanten Akteure und welche Partizipationsformate eignen sich für ihre Einbindung? Wie stark sollen und wollen Praxisakteure an Co-Design- und Co-Produktionsprozessen beteiligt werden? $\mathrm{Zu}$ welchem Zeitpunkt, in welchen Phasen und an welchen Aktivitäten sollen und wollen sie beteiligt werden, wann ist ihre Beteiligung unabdingbar, wann nicht? Wie viel Beteiligung können sie überhaupt leisten? Welche (wissenschaftlichen) Tätigkeiten bedürfen gar (z. B. wegen Datenschutzes) gezielt der Ausklammerung von Praxisakteuren?

Auch dann, wenn die Partizipation in einem Reallabor als ein „Partizipationskontinuum“ (Meyer-Soylu et al. 2016) verstanden und konzipiert wird, welches ganz unterschiedliche Formen und Formate der Einbindung entweder punktuell oder über die gesamte Dauer des Reallabors vorsieht, darf daraus keine Art ,Pflicht zu andauernder Partizipation“ aller Akteure bei allen Themen und Aktivitäten abgeleitet werden. Dies gilt auch dann, wenn die verschiedenen Formate aufeinander aufbauen oder ineinandergreifen. Vielmehr muss die Einbindung der verschiedenen Praxisakteure wohlüberlegt, wohldosiert und akzentuiert gestaltet werden - und situationsabhängig punktuell auch bewusst unterbleiben.

\section{Literatur}

Eckart, J., Ley, A., Häußler, E., \& Erl, Th. (2018). Leitfragen für die Gestaltung von Partizipationsprozessen in Reallaboren. In R. Defila \& A. Di Giulio (Hrsg.), Transdisziplinär und transformativ forschen. Eine Methodensammlung (S. 105-135). Wiesbaden: Springer VS.

Meyer-Soylu, S., Parodi, O., Trenks, H., \& Seebacher, A. (2016). Das Reallabor als Partizipationskontinuum. Erfahrungen aus dem Quartier Zukunft und Reallabor 131 in Karlsruhe. Technikfolgenabschätzung - Theorie und Praxis, 25 (3), (S. 31-40).

Selle, K. (2011). „Particitainment ${ }^{\star}$ - oder: Beteiligen wir uns zu Tode?“. PND online III, (S. 1-19). doi: 10.18154/RWTH-CONV-020780. http://www.planung-neu-denken. de/images/stories/pnd/dokumente/3_2011/selle_particitainment.pdf. Zugegriffen am 12.01.2018. 


\section{Die zwei weiteren Partizipationsmythen in diesem Buch}

Alcántara, S., Quint, A., \& Seebacher, A. (2018). Der Partizipationsmythos „Partizipation in Reallaboren muss repräsentativ sein“. In R. Defila \& A. Di Giulio (Hrsg.), Transdisziplinär und transformativ forschen. Eine Methodensammlung (S. 137-141). Wiesbaden: Springer VS.

Quint, A., Alcántara, S., \& Seebacher, A. (2018). Der Partizipationsmythos „Partizipation in Reallaboren ist per se transparent und muss es auch sein“". In R. Defila \& A. Di Giulio (Hrsg.), Transdisziplinär und transformativ forschen. Eine Methodensammlung (S. 69-73). Wiesbaden: Springer VS.

Open Access Dieses Kapitel wird unter der Creative Commons Namensnennung 4.0 International Lizenz (http://creativecommons.org/licenses/by/4.0/deed.de) veröffentlicht, welche die Nutzung, Vervielfältigung, Bearbeitung, Verbreitung und Wiedergabe in jeglichem Medium und Format erlaubt, sofern Sie den/die ursprünglichen Autor(en) und die Quelle ordnungsgemäß nennen, einen Link zur Creative Commons Lizenz beifügen und angeben, ob Änderungen vorgenommen wurden.

Die in diesem Kapitel enthaltenen Bilder und sonstiges Drittmaterial unterliegen ebenfalls der genannten Creative Commons Lizenz, sofern sich aus der Abbildungslegende nichts anderes ergibt. Sofern das betreffende Material nicht unter der genannten Creative Commons Lizenz steht und die betreffende Handlung nicht nach gesetzlichen Vorschriften erlaubt ist, ist für die oben aufgeführten Weiterverwendungen des Materials die Einwilligung des jeweiligen Rechteinhabers einzuholen. 